InnOvaciOnes de NegOciOs 16(32): 271-303

(c) 2019 UANL, Impreso en México (ISSN: 2007-1191)

Recepción: 7 Junio 2019 Aceptación: 18 Junio 2019

\title{
Los efectos de los rezagos temporales en las estrategias de operación de alta frecuencia en el mercado FOREX (The effects of temporary lags in the strategies of high frequency operation in FOREX market)
}

\author{
Luis Miguel Cruz Lázaro, Felipe A. Pérez Sosa y Jesús Abraham Martínez \\ Ceballos \\ Universidad Autónoma de Querétaro \\ corintio29@hotmail.com
}

\begin{abstract}
The aim of this work is to analyze the possible impact of time delays between the order and the execution of the operations in the FOREX market, made by High Frequency Trading strategies. This, because the use of such High Frequency Trading operations is increasing their volume in the financial markets, but their actual effects still have not been studied enough. To this end, we carried out a high frequency trading model, with which we simulated time delays, with the purpose to statistically evaluate the impacts of such anomalies in the final positions of the investments. We found that the presence of delays amongst the orders and their executions have a significant impact, which may derivate into losses. Therefore, we concluded that is important to test the technological infrastructure for High Frequency Trading, before their implementation, in order to identify possible faults and negative impacts.
\end{abstract}

Key words: High Frequency Trading, FOREX, Time delays.

JEL: O33, G15, O39.

Resumen: El objetivo de esta investigación es analizar el impacto que pueden tener los rezagos temporales entre la emisión y la ejecución de las órdenes en el mercado FOREX, mediante estrategias de alta frecuencia. Esto, debido a que el uso de estrategias de alta frecuencia tiene un importante volumen de participación en los mercados financieros, pero sus efectos aún no han sido estudiados en su totalidad. Para este fin, se elaboró un modelo de trading de alta frecuencia con el que se simularon

Los efectos de los rezagos temporales 
rezagos temporales, con el objeto de evaluar estadísticamente el impacto de estas anomalías en las posiciones finales de los inversionistas. Se encontró que la existencia de rezagos entre las órdenes y su ejecución, tienen un impacto significativo, el cual, incluso puede producir pérdidas. De tal forma, se concluyó que es importante revisar los sistemas de operación de alta frecuencia antes de implementarlos, buscando evitar fallas y sus posibles impactos negativos.

Palabras clave: Estrategias de operación de alta frecuencia, Mercado FOREX, Rezagos temporales.

\section{Introducción}

El mercado cambiario es el sitio en donde se efectúa la compra y venta de una moneda respecto a otra; algunas de sus características son el uso de una moneda vehicular, y, que es el más grande de todos los mercados financieros (Kozikowsky, 2007; Mesén, 2010; Brealey et al., 2010; Morales y Morales, 2014; Berk y DeMarzo, 2008; Mascareñas, 2012; King et al., 2011). El desarrollo de este mercado propició que no fuera necesario un lugar físico para operar, dando lugar a la formación de un mercado global de divisas, denominado: Foreign Exchange Market 0 FOREX (Miranda y Gómez, 2017; King et al., 2011; López y Calderón, 2012; Kozikowsky, 2007).

Entre las características del FOREX, se encuentran que es descentralizado, que opera las 24 horas del día, y que es clasificado como un mercado extrabursátil (Over The Computer, OTC), ya que no tiene una sede central física en la cual se lleven a cabo sus operaciones. También, que sus operadores utilizan el internet y los portales trading, para efectuar

\section{Cruz, L., Pérez, F. \& Martínez, A.}


a través de ellos sus transacciones (Kozikowsky, 2007; King et al., 2011; Mascareñas, 2012).

El desarrollo tecnológico en el mercado FOREX ha originado dos formas de operar: el trading tradicional, en el que la ejecución de las órdenes depende del operador, y el trading algorítmico, en el que un algoritmo realiza las operaciones, sin intervención de un operador. Dentro de esta última modalidad, una estrategia de operación que ha surgido recientemente es el High Frequency Trading (HFT) o Trading de Alta Frecuencia (TAF) (Miño, 2015; King et al., 2011); el cual, se define como un sistema automatizado, que, con base en estrategias previamente diseñadas, compra y vende instrumentos financieros, ejecutando sus operaciones a una alta velocidad (Sáenz, 2012; Miño, 2015; Chlistalla, 2011; Chordia et al., 2013; Hernández y Sánchez, 2017; Huang, 2012; Jones, 2013).

Dado que el TAF es un instrumento tecnológico, se ha generado la inquietud de que sea susceptible a errores y fallas, que puedan causar daños a los mercados en donde opera, al igual que a sus usuarios. No obstante, existe una falta de investigaciones y estudios al respecto, por lo que aún se desconoce la totalidad de sus efectos. Un ejemplo de un fallo de mercado en el que se vio involucrado el TAF, es el llamado Flash Crash de 2010, en el que el índice Dow Jones cayó más de mil puntos en cuestión de minutos. Si bien, las autoridades decretaron que el TAF no tuvo una relación directa con este fenómeno, existen autores que opinan que el TAF contribuyó de manera importante a que este hecho se presentara (Chlistalla, 2011; Sáenz, 2012; Sornette y Der Becke, 2011; Kirilenko, et al., 2017; Jones, 2013).

\section{Los efectos de los rezagos temporales}


Otros ejemplos son, el evento de Knight Capital, en el que se utilizó un algoritmo TAF que contenía errores, lo que terminó por ocasionar pérdidas; cuando se usó un algoritmo defectuoso en la oferta inicial de acciones de Facebook; y los mini flash crashes, que han ocurrido en distintos mercados, como el de opciones, el de futuros y el FOREX (Jones, 2013; Sornette y Der Becke, 2011). En México, un caso importante de riesgo tecnológico es el ataque a los bancos conectados al Sistema de Pagos Electrónicos Interbancarios (SPEI), en 2018, lo que también ocasiono un retraso en las transacciones de los clientes de las instituciones bancarias atacadas (Banco de México, BANXICO, 2018 a).

Por estas razones, el objetivo de este trabajo es analizar el impacto que pueden tener los rezagos temporales entre la emisión y la ejecución de las órdenes en el mercado FOREX, mediante estrategias de alta frecuencia, que pueden ser ocasionados por los riesgos tecnológicos a los que se exponen los participantes de este mercado. Asimismo, se planteó como hipótesis principal que: los rezagos temporales tienen un impacto negativo en las posiciones finales obtenidas mediante estrategias de operación de alta frecuencia en el mercado FOREX. Para ello, esta investigación se estructura de la siguiente manera: En la sección II se hace una revisión de la literatura más pertinente sobre el tema de estudio, en la tercera sección se explica el marco metodológico llevado a cabo, en la cuarta sección se muestran los resultados obtenidos, y, finalmente, en la última sección se describen las conclusiones e implicaciones derivadas de la presente investigación.

Cruz, L., Pérez, F. \& Martínez, A. 


\section{Marco teórico}

El trading de Alta Frecuencia: Definiciones, características y desarrollo

El Trading de Alta Frecuencia, o TAF, puede definirse como un sistema automatizado, que, con base en estrategias previamente diseñadas, compra y vende instrumentos financieros. Sus principales características, son: la velocidad con la que se ejecutan sus operaciones, que hacen uso de otros sistemas de trading algorítmico para ejecutar sus órdenes, que no guardan posiciones al final del día, que en sus operaciones no hay participación humana y que utilizan el acceso directo al mercado (Gomber et al., 2011; Sáenz, 2012; Miño, 2015; Chlistalla, 2011; Chordia et al., 2013; Hernández y Sánchez, 2017; Huang, 2012; Jones, 2013).

El surgimiento y la implementación de los sistemas automatizados son consecuencia de los avances tecnológicos, como la inteligencia artificial, señalada por Sáenz (2012), y de los cambios en los requisitos de los mercados, particularmente la rebaja en las tarifas de operación (Chlistalla, 2011; Kaya, 2016; Gomber et al., 2011; Ontiveros et al., 2012; Huang, 2012; King et al., 2011). En este contexto, el TAF se desarrolló a partir de los avances del trading algorítmico. Su empleo comienza en 1999 y ha ido adquiriendo mayor relevancia, hasta alcanzar un papel destacado en los mercados, como se muestra en la Figura 1 (Sáenz, 2012; Miño, 2015; Ericsson y Fridholm, 2013; Jones, 2013; Rijper et al., 2010). 
Figura 1. Estimaciones de la participación del TAF en los mercados de EUA.

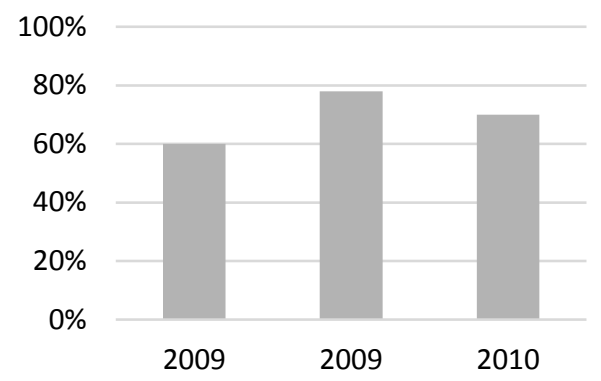

Fuente: Elaboración propia, con base en Hernández y Sánchez (2017), Ontiveros et al., (2012), Zhang (2010) y Miño (2015).

El TAF usa estrategias que ya existen, aprovechando, las tecnologías, los datos, el acceso al mercado y el enrutamiento de los pedidos, siendo la velocidad en sus operaciones su elemento más esencial, las cuales pueden ejecutarse en milisegundos. Entre las estrategias que se pueden implementar con el TAF, se hallan: el arbitraje estadístico, el cual se basa en aprovechar las fallas en la fijación de precios en los mercados, ocasionados normalmente por errores temporales; la detección de liquidez, que consiste en identificar anticipadamente los movimientos de los demás operadores; y la estrategia de creación de mercado, que busca una ganancia por medio del diferencial de precios causado por la continua compra y venta de activos (Chlistalla, 2011; Jones, 2013; Sáenz, 2012; Ontiveros et al., 2012; Gomber et al., 2011).

\section{Cruz, L., Pérez, F. \& Martínez, A.}


Por su parte, Gomber at al., (2011), mencionan que las estrategias del TAF son: proveedor electrónico de liquidez; estrategias impulsadas por rebajas, es decir, aprovechar las tarifas entre mercados; el arbitraje de latencia, que consiste en beneficiarse del acceso rápido a los mercados; y estrategias de impulso a corto plazo. Adicionalmente, Sáenz (2012) señala la estrategia de Quote Stuffing, la cual consiste en enviar grandes volúmenes de pedidos para posteriormente retirarlos rápidamente, buscando tener una ventaja frente a sus competidores debido a que estos tienen que procesar la información generada.

Consecuencias del uso del trading de alta frecuencia en los mercados

Las consecuencias del uso del TAF en los mercados aún no se han abordado de manera exhaustiva en la literatura, sin embargo, existe discusión acerca de su impacto en situaciones como el Flash Crash, de 2010, (Chlistalla, 2011; Sáenz, 2012; Sornette y Der Becke, 2011; Kirilenko, et al., 2017; Jones, 2013). Sobre este tema, Kumiega et al., (2016), al igual que Sornette y Der Becke (2011), sostienen que la interacción entre algoritmos causó un bucle que amplificó la volatilidad en el índice Dow Jones. Otros fenómenos atribuidos al TAF son los mini flash crashes, que han surgido en diversos mercados, como en el FOREX. También, el evento del Knight Capital, cuando la implementación de un TAF con fallas, ocasionó pérdidas para sus usuarios (Sornette y Der Becke, 2011; Jones, 2013).

Dentro de las consecuencias negativas que se han asociado al $\mathrm{TAF}$, se ha mencionado que esta forma de operar ha alterado las reglas en los mercados en donde participa, que la velocidad en sus ejecuciones

\section{Los efectos de los rezagos temporales}


proporciona una ventaja sobre los demás participantes, que sus altos costos de implementación son una barrera de entrada, que no proporciona liquidez a los mercados y que se beneficia de la estructura de tarifas (Chordia, et al., 2013; Sáenz, 2012; Sornette y Der Becke, 2011; Rijper et al., 2010; Jones, 2013).

Asimismo, se ha criticado su forma agresiva de operar, que pudo haber contribuido a la aparición de eventos como el Flash Crash (Kirilenko, et al., 2017). En este sentido, Huang (2012) señala que el TAF origina mayores fluctuaciones en los mercados, y Sáenz (2012) menciona que no mide las consecuencias de sus operaciones, lo que coincide con lo postulado por Zhang (2010), quien destaca que las estrategias del TAF están separadas del contexto de las empresas.

No obstante, también se han destacado algunas consecuencias positivas del TAF. Por ejemplo, Ericsson y Fridholm (2013) demuestran que el TAF ha contribuido a aumentar el volumen de operaciones en el mercado de valores sueco. A su vez, Gomber et al., (2011) opinan que el TAF ha traído una mejora en varios aspectos de las bolsas, como en la calidad de los mercados, la liquidez y favoreciendo de forma positiva en la volatilidad. Razones por las que autores como Rijper et al., (2010), Gomber, (2011) y Kaya, (2016) argumentan que el TAF es un creador de mercado que proporciona liquidez, contribuye a descubrir los precios, mejora la eficiencia del mercado, y ayuda a la reducción de los costos de transacción y la volatilidad. Asimismo, Jones (2013) sostiene que las dificultades que ha ocasionado el TAF, son similares a las originadas por el trading tradicional. 
Rezagos temporales en los modelos de trading algorítmicos y la institución del tiempo en México

De acuerdo con Sobrepere (2015), los modelos de trading algorítmicos implementados en el mercado FOREX no eliminan los errores humanos, debido a que se sustituyen los errores derivados de la psicología del operador, por otros; como errores en la programación o fallas al momento de implementar los modelos de trading algorítmicos. Uno de estos errores, que no se ha abordado suficientemente en la literatura, es el originado por los rezagos temporales entre la emisión de las órdenes y sus ejecuciones, que pueden originarse por problemas tecnológicos.

Un ejemplo de esta situación sucedió en la oferta pública inicial de Facebook, cuando un software que debía administrar las órdenes emitidas y canceladas falló por unos minutos, ocasionando pérdidas de millones de dólares entre los participantes. También, el caso de Knight Capital Group, quienes usaron un modelo de trading de alta frecuencia en la bolsa de Nueva York, el cual no probaron de manera exhaustiva antes de ponerlo en funcionamiento; $y$ una vez implementado en la bolsa, operó cerca de 45 minutos y tomó posiciones en 148 acciones de manera equivocada, generando pérdidas por $\$ 440$ millones de dólares (Jones, 2013). Otro ejemplo, es lo que sucedió en México en 2018, donde los bancos conectados al Sistemas de Pagos Electrónicos Interbancarios (SPEI), fueron atacados por un grupo de hackers con el objetivo de robarles, lo que se manifestó inicialmente con un retraso en las operaciones de los usuarios (BANXICO, 2018 a).

\section{Los efectos de los rezagos temporales}


En el caso del TAF, es pertinente recordar que la velocidad en las transacciones representa una ventaja competitiva muy importante, de manera que un posible rezago en la ejecución de las operaciones supondría un serio perjuicio. En este sentido, la hora oficial de una operación electrónica realizada en México puede certificarse a través del sellado de tiempo, emitido por el Centro Nacional de Metrología (CENAM), el cual tiene como finalidad la protección de los participantes del comercio electrónico. Cabe señalar que el CENAM tiene bajo su tutela el Patrón Nacional de Escalas de Tiempo y un reloj atómico de máxima exactitud, por lo que esta institución es la encargada de la emisión de la hora oficial (González y Zeleny, 2007; Becerril, 2013).

\section{Metodología}

La metodología que se desarrolla en esta investigación busca analizar el impacto que pueden tener los rezagos temporales entre la emisión y la ejecución de las órdenes en el mercado FOREX, mediante estrategias de alta frecuencia. Asimismo, comprobar la hipótesis principal la cual es que: los rezagos temporales tienen un impacto negativo en las posiciones finales obtenidas mediante estrategias de operación de alta frecuencia en el mercado FOREX. Para ello, se propone una investigación de tipo cuantitativo, de series de tiempo y de tipo correlacional, con base en la clasificación de Ollivier y Thompson (2017).

Para alcanzar el objetivo principal de investigación, primero se desarrolla un modelo de trading de alta frecuencia (TAF), considerando dos etapas: la primera consiste en analizar el mercado y emitir la orden correspondiente (compra, venta o mantener); y la segunda es la que

\section{Cruz, L., Pérez, F. \& Martínez, A.}


ejecuta las órdenes. El modelo de TAF se diseñará para que compre divisas cuando el tipo de cambio baje y las venderá cuando el tipo de cambio suba, por ello, la estrategia que emplea el modelo del TAF de esta investigación se considera como el arbitraje estadístico en su modalidad de alta frecuencia (Chlistalla, 2011; Jones, 2013; Gomber at al., 2011).

Para elaborar la primera parte del modelo de TAF, se utiliza de base la desviación estándar móvil muestral con una $n=14$ días sobre los logaritmos naturales de los tipos de cambio. Se opta por este parámetro, porque al emplearlo el modelo de TAF podrá analizar el grado de dispersión del tipo de cambio y esta información se utilizará como referencia para emitir la orden de compra, venta o mantener. Asimismo, el valor de la desviación estándar móvil muestral debe ser pequeña, ya que así el modelo reaccionara ante menores variaciones del tipo de cambio y por ello se considerará como un modelo de TAF. La fórmula para estimar la desviación estándar móvil muestral surge a partir de la Ecuación 1, la cual se emplea para calcular la desviación estándar muestral (Sáez, 2012; Anderson, et al. 2008). La diferencia entre la desviación estándar móvil muestral con la desviación estándar muestral, es que conforme avance el número de periodos, su estimación se va recorriendo.

$$
s_{n-1}=\sqrt[2]{\frac{\sum(x-\bar{x})^{2}}{n-1}}
$$

Donde:

Los efectos de los rezagos temporales 
282

$$
\begin{aligned}
& s_{n-1}=\text { Desviación estándar } \\
& \frac{\sum(x-\bar{x})^{2}}{n-1}=\text { Varianza } \\
& x=\text { Valor de la observación } \\
& \bar{x}=\text { Media muestral } \\
& n=\text { El número de las observaciones }
\end{aligned}
$$

Se utiliza una desviación estándar móvil muestral de .5, asumiendo que al usar está desviación estándar móvil muestral se emplea un modelo de TAF que emplea la estrategia de arbitraje estadístico en su modalidad de alta frecuencia. Como se indicó, la desviación estándar móvil muestral es el parámetro que se utiliza para emitir la orden de compra, venta o mantener. Usando de base la comparación, entre la desviación estándar móvil muestral establecida de referencia, y la diferencia de la cotización del tipo de cambio del día que se examina con el del día anterior, se desarrolla el criterio de decisión. En la Figura 2, se muestra el diagrama de flujo del criterio de decisión que se emplea en el modelo de TAF. 
Figura 2. Diagrama de flujo del criterio de decisión del modelo TAF

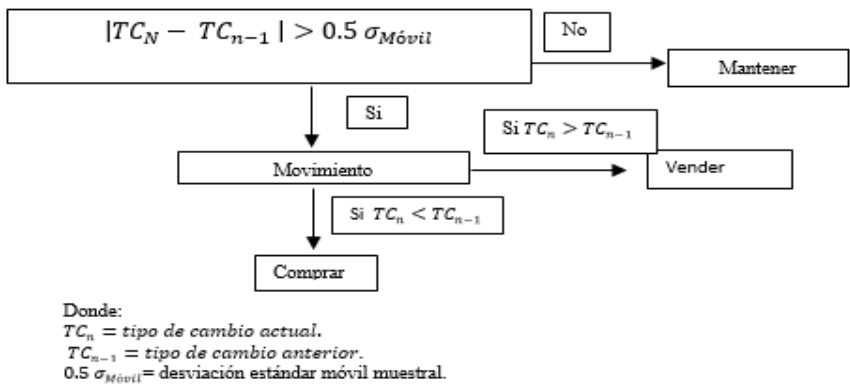

Fuente: Elaboración propia.

La expresión matemática del criterio de decisión que emplea el modelo de TAF, se desarrolla en las Ecuaciones 2, 3, 4 y 5.

Función de movimiento.

Dado que $\left|T C_{n}-T C_{n-1}\right|<0.5 \sigma_{m}$

$f(Z)=0$

Donde:

$T C_{n}=$ Tipo de cambio en el periodo actual.

$T C_{n-1}=$ Tipo de cambio del periodo anterior.

$0.5 \sigma_{m}=$ Desviación estándar móvil muestral de .5 .

$f(Z)=$ Variable aleatoria

$0=$ Mantener posición. 
Posteriormente en la Ecuación 3 se plantea.

Dado que $\left|T C_{n}-T C_{n-1}\right|>0.5 \sigma_{m}$

Donde:

$T C_{n}=$ Tipo de cambio del periodo actual.

$T C_{n-1}=$ Tipo de cambio del periodo anterior.

$0.5 \sigma_{m}=$ Desviación estándar móvil muestral de .5 .

Con lo cual se define una nueva variable aleatoria, la cual se muestra en la Ecuación 4.

$$
Z=T C_{n}-T C_{n-1}
$$

Así se desarrolla la función de la nueva variable aleatoria $Z$ y que se presenta en la Ecuación 5.

$$
f(Z)=\left\{\begin{array}{ll}
-1 & Z>0 \\
1 & Z<0
\end{array}\right\}
$$

Donde:

$-1=$ vender divisas.

$1=$ comprar divisas.

Con base en la Figura 2, o en la expresión matemática del criterio de decisión, que se desarrolla en las Ecuaciones 2, 3, 4 y 5, se crea el 
criterio de decisión del modelo de TAF. Utilizando este criterio de decisión, se crea la primera parte del modelo de TAF. Una vez que la primera parte del modelo de TAF emita la orden, la segunda parte del modelo del TAF la ejecutara. Por último, con el objeto de quitar el ruido que provoquen alteraciones en el análisis, la primera etapa del TAF, trabaja con los logaritmos naturales de los tipos de cambio, y, la segunda etapa ejecutara las órdenes sobre el tipo de cambio. En la Figura 3, se muestra el diagrama de flujo del modelo de TAF que se desarrolla, donde se aprecia la integración de las dos partes antes descritas.

Figura 3. Diagrama de flujo de la estructura del trading de alta frecuencia, con las dos partes integradas

- Se emplea logaritmo natural $\left(\frac{\mathrm{n}}{n-1}\right)$ (donde $\mathrm{n}=$ dato actual), para obtener el logaritmo natural.

- Se empleó una media móvil muestral de 14 días.

- Se empleó una desviación estándar móvil muestral de 14 días.

- Se calcula la diferencia entre $(n, n-1)$.

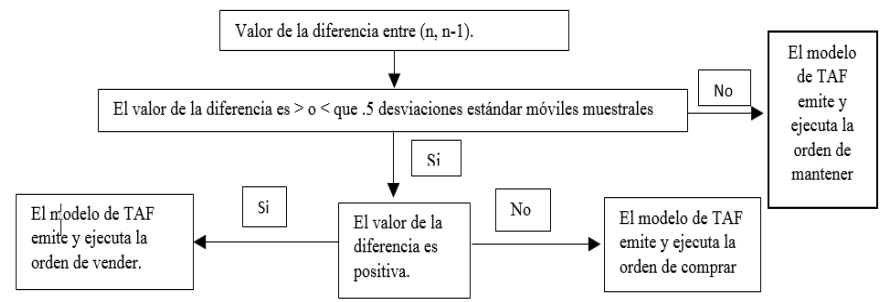

Fuente: Elaboración propia.

Posteriormente, se simulan mil escenarios a partir de una base de datos del tipo de cambio FIX peso-dólar con una frecuencia diaria, la cual se obtiene del sitio web del Banco de México. En esta investigación se emplea una base de datos que comprende del 12 de octubre de 1991, al

\section{Los efectos de los rezagos temporales}


24 de enero del 2018, la cual se obtuvo de sitio web del Banco de México (BANXICO). La cantidad de observaciones en total son, 6585 datos, correspondiente a 27 años. El hecho de que sea por día no perjudica los resultados obtenidos, basados en la propiedad escalante planteada por el análisis fractal, que indica que los riesgos y las desviaciones en los precios, mantienen sus propiedades estadísticas en diferentes frecuencias de observaciones (Mandelbrot y Hudson, 2010).

A partir de la base de datos, se simulan mil escenarios, con el fin de que los resultados obtenidos sean representativos, y abarquen los escenarios que pudieran ser atípicos. En la Figura 4, se muestra el procedimiento usado para obtener las simulaciones, donde, con el objeto de respetar la temporalidad de los datos y evitar las fluctuaciones que provoquen ruido, se extraen de forma secuencial los 14 datos por año.

Figura 4. Diagrama de flujo del procedimiento utilizado para obtener los mil escenarios

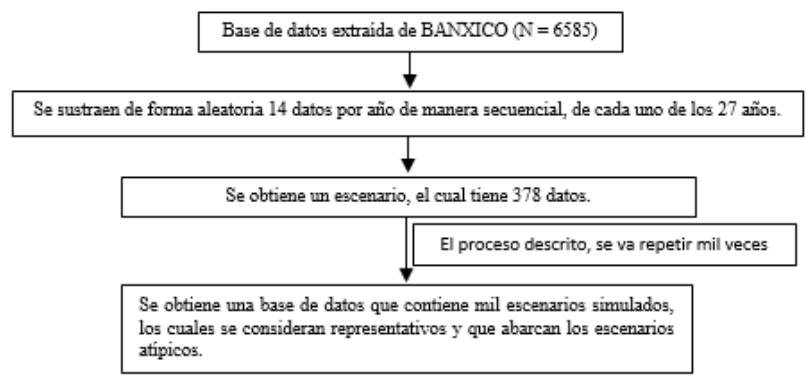

Fuente: Elaboración propia.

Cruz, L., Pérez, F. \& Martínez, A. 
Con el modelo de TAF y los mil escenarios simulados, ahora sigue generar las bases de datos de las posiciones finales obtenidas mediante estrategias de alta frecuencia, de los escenarios con rezagos temporales y sin rezagos temporales, con base en el procedimiento mostrado en la Figura 5; ajustando el número de observaciones en cada muestra, a fin de que las dos tengan la misma temporalidad y cantidad de órdenes ejecutadas, y asumiendo que no existen costos de transacción.

Figura 5. Diagrama de flujo del procedimiento utilizado para obtener la base de datos de las posiciones finales

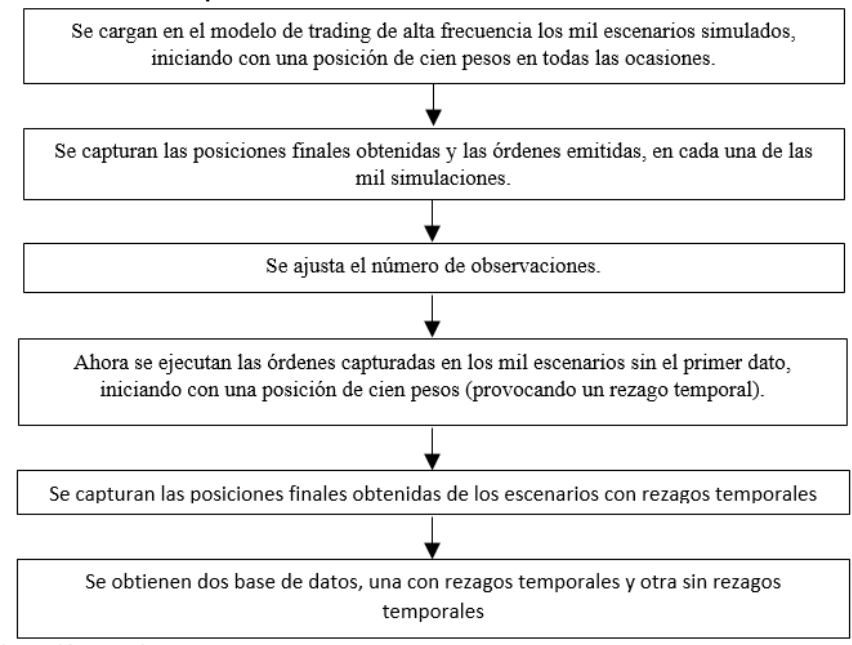

Fuente: Elaboración propia.

Los efectos de los rezagos temporales 
Además, con el objeto de analizar y comparar las posiciones finales obtenidas mediante estrategias de alta frecuencia, de los escenarios con rezagos temporales y sin rezagos temporales, se emplean la media poblacional, la mediana, la desviación estándar poblacional, y se calculan las distribuciones de frecuencias para los dos escenarios. Por último, para confirmar que sean estadísticamente diferentes las posiciones finales obtenidas mediante estrategias de alta frecuencia, de los escenarios con rezagos temporales y sin rezagos temporales, se examinan utilizando la prueba de hipótesis de dos colas para una distribución normal o Z, se optó por esta prueba, debido a la cantidad de datos de la muestra, para realizarla se utilizó el procedimiento descrito por Anderson, et al., (2008). El procedimiento, consiste en calcular la media y la desviación estándar poblacional, y, posteriormente se formulan las hipótesis de acuerdo con la Ecuación 6.

$$
\begin{aligned}
& H_{0}: \mu=\mu_{0} \\
& H_{1}: \mu \neq \mu_{0}
\end{aligned}
$$

Donde:

$\mu=$ La media base de comparación.

$\mu_{0}=$ Es la media muestral contra la cual se compara $\mu$.

Con base en la Ecuación 6, se propone la hipótesis nula $\left(H_{0}\right)$, la cual es, que la media de los escenarios con rezagos temporales es igual a la media de los escenarios sin rezagos temporales, mientras que la hipótesis alternativa $\left(H_{1}\right)$, es que son diferentes. Para aceptar la hipótesis

Cruz, L., Pérez, F. \& Martínez, A. 
nula $\left(H_{0}\right)$ y rechazar la hipótesis alternativa $\left(H_{1}\right), 0$, aceptar hipótesis alternativa $\left(H_{1}\right)$ y rechazar hipótesis nula $\left(H_{0}\right)$, se emplea la Ecuación 7.

$$
Z=\frac{\bar{x}-\mu_{0}}{\sigma / \sqrt{n}}
$$

Donde:

$\bar{x}=$ La media que toma como referencia para la prueba de hipótesis.

$\mu_{0}=$ La media con la que se está comparando.

$\sigma=$ Desviación estándar poblacional.

$n=$ Tamaño de la población.

Posteriormente, se localiza el valor crítico, para ello, se busca el valor correspondiente en las tablas de distribución normal o $Z$ con un valor $\alpha=.025$, debido a que la prueba es de dos colas. Ya con el valor crítico obtenido, a continuación, se comparan el valor del estadístico de prueba obtenido con el valor crítico, de acuerdo con el criterio de decisión mostrado en la Ecuación 8 (Anderson, et. al., 2008).

Criterio de decisión, rechazar la $H_{0}$ si: $z$

$$
\leq-Z \alpha / 2 \text { o } z \geq Z \alpha / 2
$$

Donde: 
$z=$ Valor del estadístico de prueba.

$-Z \alpha / 2=$ Valor crítico (buscado en las tablas de distribución normal).

$Z \alpha / 2=$ Valor crítico (buscado en las tablas de una distribución normal).

Con base en el criterio de decisión mostrado en la Ecuación 7, se aceptan 0 se rechazan las hipótesis planteadas. Con la prueba de hipótesis para una distribución normal de dos colas, se podrá determinar si son 0 no son estadísticamente diferentes las medias de los dos escenarios, con un $\alpha=.05$, esto es, con un nivel de significancia del $95 \%$.

\section{Resultados}

Con el fin de lograr el objetivo principal de la investigación, que es analizar el impacto que pueden tener los rezagos temporales entre la emisión y la ejecución de las órdenes en el mercado FOREX, mediante estrategias de alta frecuencia; se desarrolló la metodología explicada en la sección anterior. Para ello, primero se creó el modelo de trading de alta frecuencia, y, posteriormente, se simularon los mil escenarios los cuales se muestran en la Figura 6.

Cruz, L., Pérez, F. \& Martínez, A. 
Figura 6. Mil escenarios simulados

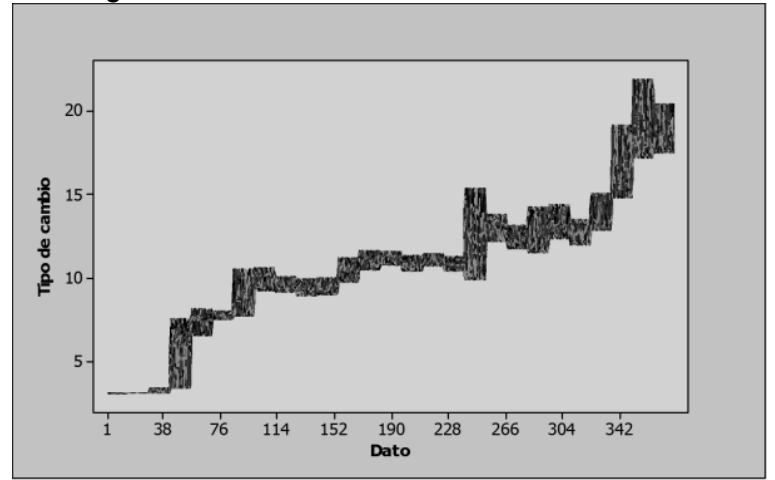

Fuente: Elaboración propia con base en BANXICO (2018 b).

Con base en los mil escenarios simulados que se muestran en la Figura 6 y en el modelo de TAF desarrollado, se simulan los rezagos temporales, como se describió en la metodología. En ambos escenarios se comenzó con una posición inicial de cien pesos, y, se capturaron las posiciones finales obtenidas, como se indicó en la metodología. Una vez creada las dos bases de datos de los escenarios, se procedió a analizarlas y compararlas con base en el procedimiento descrito en la metodología, por lo cual, se procedió a estimar la media poblacional, la mediana y la desviación estándar poblacional, además, se localizaron su valor máximo y mínimo, los resultados obtenidos se muestran en la Tabla 1. 
Tabla 1. Medidas de tendencia central en escenarios con y sin rezagos temporales

\begin{tabular}{cccccc}
\hline $\begin{array}{c}\text { Escenarios donde } \\
\text { se implementó el } \\
\text { trading de alta } \\
\text { frecuencia }\end{array}$ & $\begin{array}{c}\text { Media } \\
\text { poblacion } \\
\text { al de las } \\
\text { posicione } \\
\text { s finales } \\
\text { obtenidas }\end{array}$ & $\begin{array}{c}\text { Desviación } \\
\text { estándar } \\
\text { poblacional } \\
\text { de las } \\
\text { posiciones } \\
\text { finales } \\
\text { obtenidas }\end{array}$ & $\begin{array}{c}\text { Mediana de } \\
\text { las } \\
\text { posiciones } \\
\text { finales } \\
\text { obtenidas }\end{array}$ & $\begin{array}{c}\text { Máximo } \\
\text { valor } \\
\text { obtenido de } \\
\text { las } \\
\text { posiciones } \\
\text { finales }\end{array}$ & $\begin{array}{c}\text { Mínimo } \\
\text { valor } \\
\text { obtenido } \\
\text { de las } \\
\text { posicione } \\
\text { s finales }\end{array}$ \\
\hline $\begin{array}{c}\text { Con rezagos } \\
\text { temporales }\end{array}$ & $\$ 431.36$ & $\$ 542.077$ & $\$ 278.83$ & $\$ 6,348.31$ & $\$ 2.14$ \\
$\begin{array}{c}\text { Sin rezagos } \\
\text { temporales }\end{array}$ & $\$ 3,816.04$ & $\$ 4,907.13$ & $\$ 2,394.91$ & $\$ 77,440.29$ & $\$ 210.54$
\end{tabular}

Fuente: Elaboración propia.

En la Tabla 1, se observa el efecto de los rezagos temporales en las posiciones finales obtenidas mediante el modelo de TAF, por ejemplo, en los escenarios sin rezagos temporales la media poblacional es de $\$ 3,816.04$ pesos, en cambio cuando hay rezagos temporales, la media poblacional se contrae de manera importante hasta $\$ 431.36$ pesos, dicho de otra manera, cuando se presentan los rezagos temporales causan una contracción en las posiciones finales obtenidas mediante el modelo de TAF. Por otro lado, en la desviación estándar poblacional y la mediana, también surgen algunos efectos, como: que en los escenarios sin rezagos temporales la desviación estándar poblacional es de $\$ 4,907.13$ pesos, por el contrario, en los escenarios con rezagos temporales fue de $\$ 542.077$ pesos, y, que el valor de la mediana en los escenarios sin

\section{Cruz, L., Pérez, F. \& Martínez, A.}


rezagos temporales es de $\$ 2,394.91$ pesos y con rezagos temporales fue de $\$ 278.83$ pesos.

Otros efectos que se observaron, fue en los valores máximos y mínimos de las posiciones finales obtenidas mediante el modelo de TAF. En los escenarios con rezagos temporales la mínima posición final fue de $\$ 2.14$ pesos, por lo cual, hay pérdidas, mientras que en los escenarios sin rezagos temporales fue de $\$ 210.54$ pesos. En las posiciones finales máximas encontradas, se halló que, en los escenarios sin rezagos temporales fue de $\$ 77,440.29$ pesos y con rezagos temporales, este se contrajo hasta $\$ 6,348.31$ pesos. De acuerdo con los resultados presentados en esta sección, los rezagos temporales entre la emisión y la ejecución de las órdenes, tienen efectos negativos en las posiciones finales obtenidas mediante el modelo de TAF.

Distribuciones de frecuencia de las posiciones finales obtenidas mediante el modelo de TAF, en los escenarios con rezagos temporales y sin rezagos temporales

Las posiciones finales obtenidas mediante estrategias de alta frecuencia, en cada uno de los escenarios con rezagos temporales, se exponen en la Figura 7, donde se observa, que el valor máximo corresponde al escenario 909, el cual obtuvo un valor de $\$ 6,348.31$ pesos. Por otro lado, en la misma Figura 7 se presentan las posiciones finales capturadas de los escenarios sin rezagos temporales, en la que se observa que la posición máxima la obtuvo en el escenario 489 y que tiene un valor de $\$ 77,440.29$ pesos.

\section{Los efectos de los rezagos temporales}


Figura 7. Gráfico de las posiciones finales obtenidas mediante estrategias de alta frecuencia
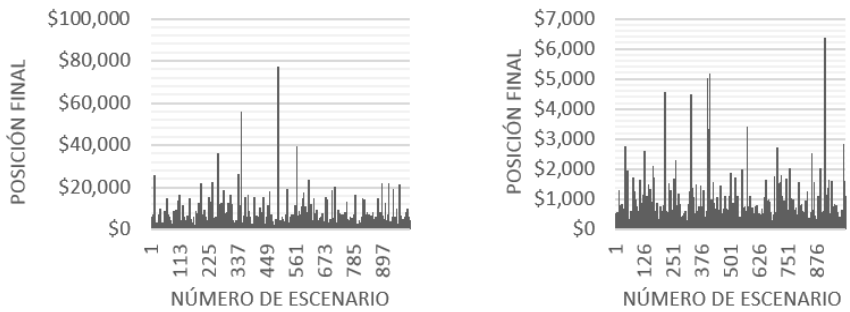

Fuente: Elaboración propia.

Basándose en las posiciones finales obtenidas mediante el modelo de TAF en los escenarios con rezagos temporales y sin rezagos temporales, y como se señaló en la metodología, se crearon los gráficos de distribución de frecuencia correspondiente a cada escenario los cuales se exponen la Figura 8.

Figura 8. Gráfico de la frecuencia de las posiciones finales obtenidas mediante el modelo de TAF
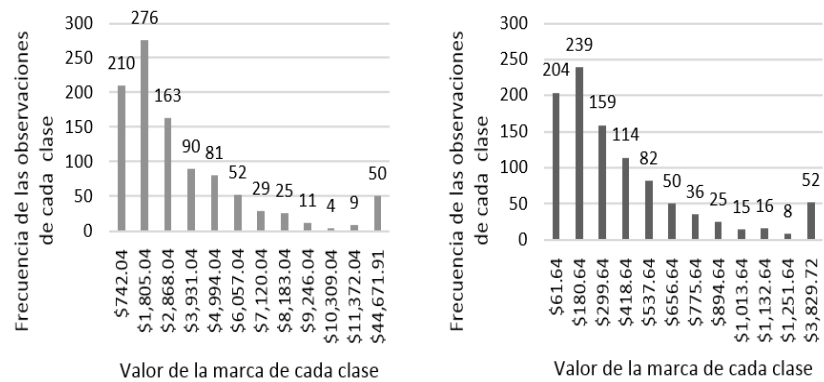

Fuente: Elaboración propia.

$$
\text { Cruz, L., Pérez, F. \& Martínez, A. }
$$


Con base en el gráfico de los escenarios con rezagos temporales que se muestra en la Figura 8 , se aprecia que la clase dos es la que agrupa más datos con 239 observaciones, equivalente a un $24 \%$, de acuerdo con la frecuencia relativa y que tiene un valor de la marca de $\$ 180.64$ pesos, también, las clases uno y dos contienen el $44 \%$ de los datos, de acuerdo con la frecuencia relativa acumulada, asimismo, se observa que la clase uno la cual contiene 204 datos, tiene un valor de la marca de $\$ 61.64$ pesos, es decir, el valor de la marca presenta pérdidas, lo que concuerda con la información mostrada en la Tabla 1. En cambio, en los escenarios sin rezagos temporales que también se muestra en la Figura 8, se observa que la clase dos concentra la mayoría de las observaciones con 276 datos, que, de acuerdo a su frecuencia relativa, equivale a un $28 \%$, y el valor de su marca es de $\$ 1,805.04$ pesos, también, que el $49 \%$ de los datos se agrupan en la clase uno y dos, además, que ninguna de sus clases tiene un valor de la marca que tenga pérdidas.

Como se destaca en los gráficos de la Figura 7 , se observa un comportamiento diferente en los dos escenarios, también, los rangos de las máximas posiciones finales obtenidas mediante el modelo de TAF son distintos, debido a que, en la Figura 7 hay posiciones finales que superan los $\$ 50$ mil pesos o $\$ 70$ mil pesos, que corresponden a los escenarios donde no hay rezagos temporales, en cambio, en los escenarios donde hay rezagos temporales la posición final más alta supera solo $\$ 6$ mil pesos, y, que se puede apreciar en la Figura 7.

En la sección de las distribuciones de frecuencia, no se aprecia una diferencia tan significativa en el comportamiento de las distribuciones

\section{Los efectos de los rezagos temporales}


de frecuencia de los dos escenarios. Sin embargo, en el valor que tienen las marcas de cada una de las clases, se presentan diferencias importantes entre los dos escenarios, y que se pueden verificar en la Figura 8, un ejemplo: es que, en la clase dos de los escenarios que no tuvieron rezagos temporales su marca es de $\$ 1,805.04$ pesos, en cambio la misma clase, pero en los escenarios con rezagos temporales el valor de su marca es de $\$ 180.64$ pesos, que es una diferencia significativa, estas diferencias se repiten en el valor de las marcas de todas las clases.

Resultados de la prueba de hipótesis

De acuerdo con la metodología, para demostrar si son estadísticamente diferentes las posiciones finales obtenidas mediante el modelo del TAF, en los escenarios con rezagos temporales y sin rezagos temporales, se aplicó una prueba de hipótesis de dos colas para una distribución normal o Z. En la Tabla 2 se exponen los resultados de la prueba de hipótesis de dos colas para una distribución normal o $Z$ de los escenarios con rezagos temporales y sin rezagos temporales, también, en la Tabla 3 se exhibe el criterio de decisión.

Tabla 2. Resultados de la prueba de hipótesis de dos colas para una distribución normal

\begin{tabular}{ccc}
\hline Hipótesis & Valor crítico obtenido & Estadístico de prueba \\
\hline$H_{o}=\$ 3,816.04$ & 197.4497591 & 1.959963985 \\
$H_{1} \neq \$ 3,816.04$ & & \\
\hline
\end{tabular}

Fuente: Elaboración propia.

Cruz, L., Pérez, F. \& Martínez, A. 
Tabla 3. Criterio de decisión correspondiente a las posiciones finales obtenidas mediante el modelo de TAF

Fuente: Elaboración propia.

\begin{tabular}{ll}
\hline Valor crítico obtenido & Estadístico de prueba \\
\hline $197.4497591 \geq$ & 1.959963985 \\
\hline aboración propia. &
\end{tabular}

De acuerdo con los resultados mostrados en la Tabla 2 y al criterio de decisión expuesto en la Tabla 3, se observa que el valor crítico es mayor que el estadístico de prueba, por lo tanto, se rechaza la hipótesis nula $\left(H_{o}\right)$ y se acepta la hipótesis alternativa $\left(H_{1}\right)$. Con base en lo anterior, se puede afirmar que las posiciones finales obtenidas mediante el modelo de TAF, en los escenarios con rezagos temporales, son estadísticamente diferentes a las posiciones finales obtenidas mediante el modelo de TAF, en los escenarios sin rezagos temporales, con un nivel de significancia del $95 \%$.

Comparación de los escenarios con rezagos temporales y sin rezagos temporales, para la toma de decisiones

A partir de los resultados obtenidos, se comprueba que el impacto de los rezagos temporales en los modelos de TAF usados en los mercados cambiarios, es perjudicial, ya que impacta de forma negativa en las posiciones finales. Por lo cual, se demuestra que, para los usuarios de las estrategias de operación de alta frecuencia, para, los cuales, es importante el tiempo en sus operaciones. Una falla derivado de un rezago temporal, entre las emisiones de sus órdenes y la ejecución de las mismas, les ocasionará una disminución en sus posiciones finales, y, de

\section{Los efectos de los rezagos temporales}


acuerdo a las simulaciones, en algunos casos pérdidas. Por lo tanto, los resultados permiten afirmar que los usuarios de las estrategias de operación de alta frecuencia, deben revisar sus mecanismos de emisión y ejecución de las órdenes, ya que, si se presenta una falla, les ocasionara un impacto negativo.

\section{Conclusiones}

El objetivo principal de la presente investigación, fue analizar el impacto que pueden tener los rezagos temporales entre la emisión y la ejecución de las órdenes en el mercado FOREX, mediante estrategias de alta frecuencia. Este objetivo se planteó, basándose en el progreso que han tenido las tecnologías en los mercados financieros, lo cual ha causado la aparición de nuevos riesgos y problemas, que no han sido investigados a profundidad.

Para este fin, se creó un modelo de trading de alta frecuencia (TAF) que emplea la estrategia de arbitraje estadístico en su modalidad de alta frecuencia. Para su diseño se utilizó la desviación estándar móvil muestral de .5, la cual se asumió como una estrategia de alta frecuencia. Simultáneamente, se simularon mil escenarios extraídos de una base de datos del tipo de cambio FIX, la cual se obtuvo del sitio web el Banco de México, que comprendió un periodo de 27 años, abarcando de 1991 a 2018. Se decidió que fueran mil escenarios, con el objeto de que los resultados incluyeran los escenarios atípicos. Estos escenarios se emplearon para simular los rezagos temporales.

Como parte de la investigación, se simularon los rezagos temporales entre la emisión de las órdenes y su ejecución, empleando el

\section{Cruz, L., Pérez, F. \& Martínez, A.}


modelo de TAF en los mil escenarios simulados. Con ese fin, se registraron las órdenes que emitió el modelo de TAF, y a continuación estas órdenes se ejecutaron en los mismos mil escenarios, sin embargo, se les quito el primer dato, por lo tanto, se crearon los rezagos temporales entre la emisión de las órdenes y su ejecución.

Con base en los resultados de la estadística descriptiva, se halló que en los escenarios donde se presentaron rezagos temporales, en promedio, causaron una contracción en las posiciones finales obtenidas mediante el modelo de TAF, encontrando escenarios donde hubo pérdidas. Por otra parte, empleando la prueba de hipótesis para una distribución normal o Z, se pudo comprobar que los dos escenarios son estadísticamente diferentes. La hipótesis planteada en esta investigación, fue que los rezagos temporales tienen un impacto negativo en las posiciones finales obtenidas mediante estrategias de operación de alta frecuencia en el mercado FOREX. De acuerdo con la evidencia encontrada, la hipótesis planteada se acepta, ya que se demostró que los rezagos temporales tienen un impacto negativo en las posiciones finales obtenidas mediante estrategias de alta frecuencia.

Basándose en los resultados obtenidos, se pudo concluir en esta investigación que la presencia de los rezagos temporales en las operaciones de trading de alta frecuencia, ocasionan una contracción en las posiciones finales obtenidas, y en algunas ocasiones pérdidas. Ante esto, se puede afirmar que se alcanzó el objetivo principal que se planteó en esta investigación. Lo anterior, es importante para los operadores que emplean este tipo de estrategias los cuales tienen una participación destacada en los mercados financieros, ya que, se aportan pruebas de

\section{Los efectos de los rezagos temporales}


los efectos que tendrían una falla en los modelos del TAF que provoquen rezagos temporales entre la emisión y ejecución de las ordenas.

Asimismo, se contribuye al estudio de las nuevas tecnologías, abordándolas desde la simulación de los rezagos temporales, ayudando a definir el impacto que tienen en las estrategias de operación de alta frecuencia y las implicaciones que tendrían para los usuarios de este tipo de estrategias. Como futura línea de investigación, se propone desarrollar un estudio que tome en consideración los costos en las transacciones, con el fin de comparar los resultados que se obtengan con los expuestos en esta investigación, para establecer si hay cambios, debido a que los costos de transacción podrían alterar los resultados. Otra futura línea que se plantea, es usar otro modelo de trading de alta frecuencia, que no utilice la desviación estándar móvil muestral. Por último, se propone utilizar el modelo de trading de alta frecuencia en otros mercados, como el de acciones.

\section{Referencias}

Anderson, D., Sweeney, D., \& Williams, T. (10a. Ed.) (2008). Estadística para administración y economía. México: Cenage Learning

Banco de México (2018). Puntos importantes sorbe situación actual de SPEl. Recuperado de: http://www.banxico.org.mx/inicio/banner/informacionimportante-sobre-la-situacion-del-spei/\%7B022CD9D7-11A9-68E6-D1A5965F57A23F60\%7D.pdf

Banco de México (2018). Tipo de cambio pesos por dólar E.U.A., tipo de cambio para solverntar obligaciones denominadas en moneda extranjera. Recuperado de: http://www.banxico.org.mx/Sielnternet/consultarDirectoriolnternetAction.do?a ccion $=$ consultarCuadro\&idCuadro $=$ CF102\&locale $=e s$

\section{Cruz, L., Pérez, F. \& Martínez, A.}


Becerril, I. (2013). El sello digital de tiempo: optimización del atecnología aplicada. Revista digital universitaria, 14(7). Recuperado de: http://www.revista.unam.mx/vol.14/num7/art20/

Berk, J. \& DeMarzo, P. (1ª . Ed.) (2008). Finanzas corporativas. México: Pearson.

Brealey, A. Myers, C. \& Allen, F. (9a. Ed.) (2010). Principios de finanzas corporativas. México: McGraw Hill.

Chlistalla, M. (2011). High-frequency trading, better than its reputation? Deutsche Bank Research. Recuperado de: https://secure.fia.org/ptg-downloads/dbonhft211.pdf

Chordia, T., Goyal, A., Lehmann, B., \& Saar, G. (2013). High-frequency trading. Johnson School Research Paper Series. 20. Recuperado de: http://dx.doi.org/10.2139/ssrn.2278347

Ericsson, T. \& Fridholm, P. (2013). High-frequency trading-impacts of the introduction of the INET platform on NASDAQ OMX Stockholm (Degree Thesis in Business Adminsitration). Stockholm University, School of Business, Sweden

Gomber, P., Arndt, B. Lutat, M., \& Uhle, T. (2011). High-frequency trading. Goethe Universität. Recuperado de: http://dx.doi.org/10.2139/ssrn.1858626

González, C. \& Zeleny, R. (1ª. Ed.) (2007). Metrología. Mécivo: McGraw-Hill Interamericana

Hernández, D. \& Sánchez, K. (2017). Un modelo de creación de mercado con trading de alta frecuencia. ODEON, (11), 123-142. Recuperado de: https://doi.org/10.18601/17941113.n11.06

Huang, Y. (2012). High-frequency trading, Technical report No. UCB/EECS-2012-130, University of California. Recuperado de: http://www.eecs.berkeley.edu/Pubs/TechRpts/2012/EECS-2012-130.html.

Jones, C. (2013). What do we know about high-frequency trading? Columbia business school, 13(11). Recuperado de: http://dx.doi.org/10.2139/ssrn.2236201

Kaya, O. (2016). High-frequency trading: reaching the limits. Deutsche bank research. Recuperado de: https://es.scribd.com/document/360921920/High-frequencyTrading-Reaching-the-Limits

\section{Los efectos de los rezagos temporales}


King, M., Osler, C., \& Rime, D. (2011). Foreign exchange market structure, players and evolution. Norges Bank. Recuperado de: https://www.norgesbank.no/en/Published/Papers/Working-Papers/2011/WP-201110/

Kirilenki, A., Kyle, A., Samadi, M., \& Tuzun, T. (2017). The flash crash: the impact of high-frequency trading on an electronic market. Journal of finance, 72(3), 967998. Recuperado de: https://doi.org/10.1111/jofi.12498

Kozikowsky, Z. (3 ${ }^{\text {a }}$ Ed.) (2007). Finanzas internatcionales. México: McGraw-Hill

Kumiega, A. Sterijevski. G, \& Van Vliet, B. (2016). Beyond the flash crash: systemic risk, reliability, and high-frequency financial markets. Journal of trading, 11(2), 7183. Recuperado de: https://ssrn.com/abstract=2712768

Lópex, P., \& Calderón, C. (2012). Caracterización de la formación de la estrategia en organizaciones del mercado FOREX. Révista Facultad de Ciencias Económicas: Investigación y reflexión, 20(1), 37-56. Recuperado de: http://www.scielo.org.co/scielo.php?script=sci_arttext\&pid=S0121$68052012000100004 \&$ Ing=en\&tlng=es

Mandelbrot, B., \& Hudson, R. (2010). Fractales y finanzas. Una aproximación matemática a los mercados: arriesgar, perder y ganar. España: Tusquets Editores

Mascareñas, J. (2012). Mercado de divisas. Universidad Complutense de Madrid. Recuperado de: http://webs.ucm.es/info/jmas/mon/22.pdf o ISSN: 1988-1878

Mesén, V. (2010). Contabilización de contratos, de futuros, opciones, FORWARDS Y SWAPS. Tec empresarial, 4(1), 42-48

Miño, J. (2015). Análisis y comparativa de los sistemas automáticos de trading frente al trading discrecional. Universidad Pontificia Comillas ICAI-ICADE. Facultad de ciencias económicas y empresariales. Recuperado de: https://repositorio.comillas.edu/xmlui/bitstream/handle/11531/3703/TFG0011 00.pdf?sequence $=1$

Miranda, S., \& Gómez, M. (2017). Mercado de divisas global y la posición de México 2016. Economía actual, (1). Recuperado de: http://web.uaemex.mx/feconomia/Publicaciones/e1001/EA2017101_Sergio_Rocio.pdf 
Morales, A., \& Morales, J. (1 ${ }^{\text {a }}$. Ed.) (2014). Planeación financiera. México: Patria

Ollivier J., \& Thompson, P. (1ª Ed.) (2017). Guía para elaborar trabajos de investigación. Chihuahua, México: Universidad Autónoma de Chihuahua

Ontiveros, E., Martín, Á., Navarro, M., \& Rodríguez, E. (1ª. Ed.) (2012). Las TIC y el sector financiero del futuro. Barcelona: Ariel

Rijper, T., Sprenkeler, W., \& Kip, S. (2010). High frequency trading. Optiver. Recuperado de: http://fragmentation.fidessa.com/wp-content/uploads/High-FrequencyTrading-Optiver-Position-Paper.pdf

Sáenz, G. (2012). Progress vs security, High frequency trading. Msc Computing Systems Engineering, Politecnico di Milano

Sáez, A. J. (1a. Ed.) (2012). Apuntes de estadística para ingenieros. España: Universidad de Jean

Sobrepere, M. (2015). Análisis de Modelos de Trading Algorítmico en el Mercado Forex. Universidad Pontificia ICAI-ICADE Comillas. Recuperado de: http://hdl.handle.net/11531/4468

Sornette, D., \& Der Becke, S. (2011). Crashes and High frequency trading. Swiss Finance Institute Research, 11(63). Recuperado de: http://dx.doi.org/10.2139/ssrn.1976249

Zhang, X. (2010). High-frequency trading, stock volatility, and price discovery. Yale University, Schoolg of Management. Recuperado de: http://dx.doi.org/10.2139/ssrn.1691679 